\title{
New anticoagulant coatings and hemostasis assessment tools to avoid complications with pediatric left ventricular assist devices
}

\author{
Daniel C. Leslie, $\mathrm{PhD},{ }^{\mathrm{a}}$ Anna Waterhouse, $\mathrm{PhD},{ }^{\mathrm{b}, \mathrm{c}}$ and Donald E. Ingber, $\mathrm{MD}, \mathrm{PhD}^{\mathrm{d}, \mathrm{e}, \mathrm{f}}$
}

From the ${ }^{\mathrm{a}}$ FreeFlow Medical Devices, LLC, Lancaster, Pa; ${ }^{\mathrm{b}}$ University of Sydney, Charles Perkins Center, Central Clinical School, School of Medicine, Sydney, New South Wales, Australia; ${ }^{c}$ Heart Research Institute, Newtown, New South Wales, Australia; the ${ }^{\mathrm{d}}$ Wyss Institute for Biologically Inspired Engineering at Harvard University; the ${ }^{\text {e } V a s c u l a r ~ B i o l o g y ~ P r o g r a m ~ a n d ~ D e p a r t m e n t ~ o f ~ S u r g e r y, ~ B o s t o n ~ C h i l d r e n ' s ~ H o s p i t a l ~ a n d ~ H a r v a r d ~}$ Medical School, Boston, Mass; and the ${ }^{\mathrm{f}}$ Harvard John A. Paulson School of Engineering and Applied Sciences, Harvard University, Cambridge, Mass

Supported by the Wyss Institute for Biologically Inspired Engineering at Harvard University, the Heart Research Institute, and the Discovery Early Career Researcher Award from the Australian Research Council.

D.C.L. and A.W. contributed equally to this article.

Received for publication Jan 31, 2017; accepted for publication March 16, 2017; available ahead of print May 16, 2017.

Address for reprints: Donald E. Ingber, MD, PhD, Wyss Institute at Harvard University, CLSB5, 3 Blackfan Circle, Boston, MA 02115 (E-mail: don.ingber@wyss.harvard.edu).

J Thorac Cardiovasc Surg 2017;154:1364-6

$0022-5223 / \$ 36.00$

Copyright (C) 2017 Published by Elsevier Inc. on behalf of The American Association for Thoracic Surgery http://dx.doi.org/10.1016/j.jtcvs.2017.03.149

\section{CHALLENGE: THROMBOSIS AND BLEEDING}

The only pediatric left ventricular assist device (pLVAD) specifically approved for use in children by the Food and Drug Administration is the EXCOR Pediatric VAD (Berlin Heart GmbH, Berlin, Germany). The EXCOR VAD gained approval on the basis of a clinical study in 2012 demonstrating significant survival benefit relative to extracorporeal membrane oxygenation in patents receiving it as a bridge to transplant. ${ }^{1}$ The median survival time was longer than 144 days for the pLVAD, whereas it was only 10 days in the matched extracorporeal membrane oxygenation cohort. Despite this remarkable achievement, serious adverse events occurred in most study participants on the EXCOR, including infection $(57 \%)$, stroke $(29 \%)$, and major bleeding $(46 \%)$. In the study of 48 total patients, thrombosis was observed in 43 EXCOR VADs, requiring the pump to be changed. Moreover, the recently established PediMACS registry indicates that the occurrence and type of adverse events have not changed since this pivotal trial. ${ }^{2}$ These numbers highlight the central conundrum: How do we titer anticoagulant and antiplatelet therapy when faced with concurrent thrombosis and bleeding risks?

\section{NEED FOR BETTER CLINICAL ASSESSMENT TOOLS AND ANTITHROMBOTIC DRUGS}

Managing the levels of antithrombotic agents with current clinical analysis tools is incredibly challenging in these patients. Extensive coagulopathy analysis is performed both before and after implantation of the pLVAD, and combinations of multiple anticoagulant, antiplatelet, antifibrinolytic, and procoagulant drugs are used at different stages in an

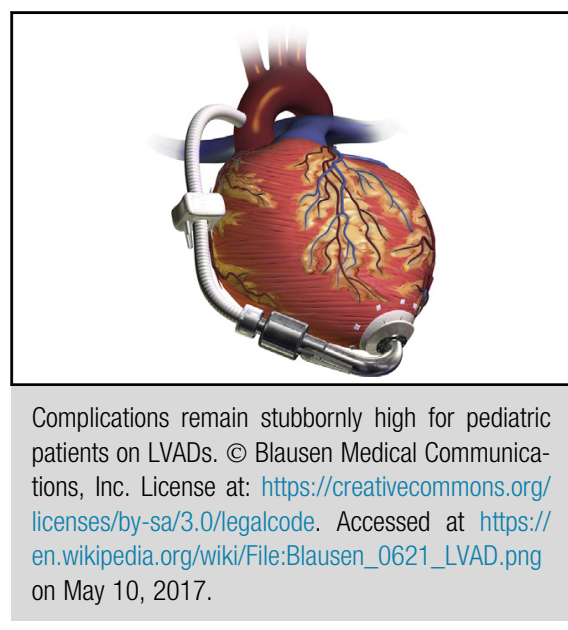

Central Message

Improved antithrombotic surface coatings and tools to assess anticoagulation and antiplatelet function offer a potential way to reduce thrombosis and bleeding events in pediatric patients with LVADs.

See Editorial Commentary page 1367.

attempt to manage the thrombotic and bleeding risks. The types of coagulopathy analysis performed and the regimens of antithrombotic drugs used vary greatly among centers, ${ }^{3}$ and despite the use of the recommended Edmonton Anticoagulation and Platelet Inhibition Protocol in pediatric patients, there remains a stubbornly high incidence of serious adverse events, including pump change in $56 \%$ of patients, major bleeding in $43 \%$, and neurologic events in $28 \%{ }^{4}$ Current clinical antithrombotic drug management is challenging for practitioners and remains ineffective for patients.

The current clinical measurements to assess platelet function and anticoagulant status include activated clotting time, activated partial thromboplastin time, partial thromboplastin time, international normalized ratio, anti-factor $\mathrm{Xa}$ levels, and thromboelastography ${ }^{5}$; however, none of these accurately predict a patient's current bleeding or thrombotic risk. ${ }^{6}$ New methods for real-time coagulopathy status monitoring are desperately needed for better management of antithrombotic therapies in complex situations, such as the case for patients with pLVADs. Recent promising approaches include the use of an ultrasound-based 
whole blood viscoelastic test that provides detailed results in approximately 10 minutes. ${ }^{6}$ It also may be helpful to take into account physiologically relevant hemodynamic forces and biologic components in coagulopathy analyses. ${ }^{5}$ For example, given that shear gradients are unavoidable in ventricular assist devices, one approach for monitoring could be a shear gradient-activated microfluidic hemostasis monitor, which evaluates coagulation and platelet activation status after a patient's blood is exposed to a series of microengineered stenotic arteriole-sized vessels. ${ }^{7}$ Interestingly, this device can be used not only in analytic laboratories but at the bedside by integrating the miniaturized microfluidic device directly into blood lines or catheters that are placed in a patient. Measurement of blood thrombosis in both static and flow environments, along with the exposure to endogenous factors or cells such as endothelium ${ }^{8}$ may finally give clinicians the tools needed to gauge blood dysfunction accurately in real time. Bringing these results quickly to the clinic is no small task, but they may provide critical insight that could greatly improve antithrombotic medication management.

The multiple symptoms and adverse events experienced by patients with pLVADs are a consequence of a variety of contributing factors, including the device materials, complex geometries, blood flow conditions, and drugs received. The complex interplay among these factors results in the activation and consumption of coagulation factors, activation of platelets, and the more recently recognized prevalence of acquired von Willebrand disease in patients with pLVADs. ${ }^{9}$ Evolving medical management therefore may include newer, more specific anticoagulant and antiplatelet drugs, such as factor XII inhibitors that inhibit materialinduced thrombosis ${ }^{10}$ and phosphoinositide 3-kinase inhibitors that decrease platelet shear activation. ${ }^{11}$ The ideal clinical scenario would be to match the results of these new analyses directly with specific recommendations for a targeted drug approach or a combination of drugs on a case-by-case basis.

\section{NEED FOR BETTER DEVICES}

Ultimately, we believe that better hemostasis assessment alone will not completely solve the thrombosis and bleeding problems in patients with pLVADs. Another way to prevent thrombosis and bleeding is to design better "stealth" devices that do not activate thrombotic responses, thereby limiting or perhaps one day eliminating the need for dangerous antithrombotic drugs altogether. Potentially improved implantable pLVADs currently being evaluated in clinical trials include the HeartMate 3 (Thoratec Corp, Pleasanton, Calif), the MVAD (HeartWare Inc, Framingham, Mass), and even a small (15 mm) implantable artificial heart (Jarvick Heart, Inc, New York, NY) for infants. ${ }^{12}$ These companies have focused on improved pump design with continuous flow. We believe, however, that a focus on improved surface coatings will further reduce the serious adverse events in patients with pLVADs.

\section{ANTITHROMBOTIC SURFACE COATINGS}

To prevent clot formation, devices are now being coated directly to prevent coagulation. The EXCOR pLVAD is coated with a CARMEDA (Carmeda AB, Upplands Väsby, Sweden) heparin-based coating, and yet thrombosis and stroke remain high despite the active anticoagulant drug immobilized directly on the surface of the pump. Surprisingly, active heparin is still bound to the surface of the device after almost a year in a patient's blood flow. ${ }^{13}$ This underscores the challenge in designing surfaces that prevent thrombosis; even coating the surface of pLVADs with a potent anticoagulant drug has not reduced the need for systemic administration of anticoagulants. ${ }^{4}$

Newer antithrombogenic coatings have been developed during the past few years that may help reduce pLVAD thrombosis in the future. A class of zwitterionic hydrophilic coatings (polymers of sulfobetaine and carboxybetaine) that are low protein binding have shown promise in reducing thrombosis on catheters in short-term dog experiments and have even gained Food and Drug Administration approval for use in catheters. ${ }^{14}$ Liquid-infused coatings $^{15-17}$ and adherent liquid coatings ${ }^{18}$ are other promising new technologies that may reduce the need for systemic anticoagulants in patients with pLVADs. Liquidinfused coatings, such as those produced with the Slippery Liquid Porous-Infused Surfaces (SLIPS) technology (SLIPS Technologies, Inc, Cambridge, Mass) permeate a porous or roughened surface with an omniphobic liquid (eg, perfluorocarbon or silicone). A related adherent liquid coating method, tethered liquid perfluorocarbon (TLP) coatings, can be used to coat smooth surfaces of existing clinically approved medical devices. These TLP coatings consist of a liquid perfluorocarbon layer that is covalently attached to the smooth surfaces of medical devices and then overlaid with another layer of freely flowing liquid perfluorocarbon. ${ }^{18}$ These TLP coatings present a smooth liquid surface in contact with blood and prevent thrombosis by stopping the adhesion of proteins, platelets, and cells to the surface of the medical devices. Provocatively, TLP-coated arteriovenous shunts remained patent in a short-term (8-hour) study in pigs without the administration of any anticoagulants. One additional potential benefit to both polysulfobetaine and liquid coatings is their resistance to biofilm and pathogen adhesion. ${ }^{14-16,18}$ It is to be hoped that preliminary results demonstrating a reduction in infection rates in mice with liquid-infused coatings ${ }^{15}$ will translate into a clinical reduction in deviceassociated infections in the future. Ultimately, the most significant impact of these low-adhesion coatings would be to help lower the amount of thrombosis in pLVADs and to reduce the number and dose of antithrombotic 
agents required to suppress bleeding events in patients with these devices.

\section{CONCLUSIONS}

With the steadily increasing use of pLVADs in children, new therapeutic avenues need to be developed to address the persistently high and varied complication rates seen clinically. There are unmet needs for improved coagulopathy monitoring, more specific anticoagulation and antiplatelet drug therapies, and improved materials and surface device coatings to reduce the disastrous thrombotic and bleeding events observed with pLVADs. With the complex nature of individual patients' profiles and device characteristics, only a combined, multifaceted approach incorporating improvements in all areas will likely lead to a major improvement in care of these desperately ill pediatric patients.

\section{Conflict of Interest Statement}

D.C.L., A.W., and D.E.I. are inventors on tethered liquid perfluorocarbon and shear-gradient-activated microfluidic hemostasis monitor patent applications. D.C.L. is employed at FreeFlow Medical Devices, LLC, which licensed the tethered liquid perfluorocarbon technology, and he holds equity in the company. D.E.I. holds equity in FreeFlow Medical Devices, LLC, and chairs its scientific advisory board. This work was supported by the Wyss Institute for Biologically Inspired Engineering at Harvard University, the Heart Research Institute, and the Discovery Early Career Researcher Award from the Australian Research Council.

\section{References}

1. Fraser CD Jr, Jaquiss RD, Rosenthal DN, Humpl T, Canter CE, Blackstone EH, et al; Berlin Heart Study Investigators. Prospective trial of a pediatric ventricular assist device. N Engl J Med. 2012;367:532-41.

2. Blume ED, Rosenthal DN, Rossano JW, Baldwin JT, Eghtesady P, Morales DL, et al; PediMACS Investigators. Outcomes of children implanted with ventricular assist devices in the United States: first analysis of the Pediatric Interagency
Registry for Mechanical Circulatory Support (PediMACS). J Heart Lung Transplant. 2016;35:578-84.

3. Moffett BS, Cabrera AG, Teruya J, Bomgaars L. Anticoagulation therapy trends in children supported by ventricular assist devices: a multi-institutional study. ASAIO J. 2014;60:211-5.

4. Steiner ME, Bomgaars LR, Massicotte MP, Berlin Heart EXCOR Pediatric VAD IDE Study Investigators. Antithrombotic therapy in a prospective trial of a pediatric ventricular assist device. ASAIO J. 2016;62:719-27.

5. Tynngård N, Lindahl TL, Ramstrom S. Assays of different aspects of haemostasis-what do they measure? Thromb J. 2015;13:8.

6. Reynolds PS, Middleton P, McCarthy H, Spiess BD. A comparison of a new ultrasound-based whole blood viscoelastic test (SEER sonorheometry) versus thromboelastography in cardiac surgery. Anesth Analg. 2016;123: 1400-7.

7. Jain A, Graveline A, Waterhouse A, Vernet A, Flaumenhaft R, Ingber DE. A shear gradient-activated microfluidic device for automated monitoring of whole blood haemostasis and platelet function. Nat Commun. 2016;7:10176.

8. Jain A, van der Meer AD, Papa AL, Barrile R, Lai A, Schlechter BL, et al. Assessment of whole blood thrombosis in a microfluidic device lined by fixed human endothelium. Biomed Microdevices. 2016;18:73-9.

9. Gossai N, Brown NM, Ameduri R, Zantek ND, Louis JS, Steiner ME. Pediatric acquired von Willebrand disease with Berlin Heart Excor ventricular assist device Support. World J Pediatr Congenit Heart Surg. 2016;7:614-8.

10. Worm M, Köhler EC, Panda R, Long A, Butler LM, Stavrou EX, et al. The factor XIIa blocking antibody 3F7: a safe anticoagulant with anti-inflammatory activities. Ann Transl Med. 2015;3:247-51.

11. Nylander S, Kull B, Björkman JA, Ulvinge JC, Oakes N, Emanuelsson BM, et al. Human target validation of phosphoinositide 3-kinase (PI3K) $\beta$ : effects on platelets and insulin sensitivity, using AZD6482 a novel PI3K $\beta$ inhibitor. J Thromb Haemost. 2012;10:2127-36.

12. Adachi I, Burki S, Zafar F, Morales DL. Pediatric ventricular assist devices. J Thorac Dis. 2015;7:2194-202.

13. Werkkala K, Jokinen JJ, Soininen L, Dellgren G, Hallhagen S, Sundberg F, et al. Clinical Durability of the CARMEDA BioActive Surface in EXCOR Ventricular Assist Device Pumps. ASAIO J. 2016;62:139-42.

14. Smith RS, Zhang Z, Bouchard M, Li J, Lapp HS, Brotske GR, et al. Vascular catheters with a nonleaching poly-sulfobetaine surface modification reduce thrombus formation and microbial attachment. Sci Transl Med. 2012;4: $153 \mathrm{ra} 132$.

15. Chen J, Howell C, Haller CA, Patel MS, Ayala P, Moravec KA, et al. An immobilized liquid interface prevents device associated bacterial infection in vivo. Biomaterials. 2017;113:80-92.

16. Epstein AK, Wong TS, Belisle RA, Boggs EM, Aizenberg J. Liquid-infused structured surfaces with exceptional anti-biofouling performance. Proc Natl Acad Sci U S A. 2012;109:13182-7.

17. Wong TS, Kang SH, Tang SK, Smythe EJ, Hatton BD, Grinthal A, et al. Bioinspired self-repairing slippery surfaces with pressure-stable omniphobicity. Nature. 2011;477:443-7.

18. Leslie DC, Waterhouse A, Berthet JB, Valentin TM, Watters AL, Jain A, et al. A bioinspired omniphobic surface coating on medical devices prevents thrombosis and biofouling. Nat Biotechnol. 2014;32:1134-40. 